\title{
Factores de riesgo para el desarrollo de toxicidad hematológica durante la aplicación de paclitaxel semanal en cáncer de mama
}

\author{
Alberto Alvarado-Miranda', Germán Calderillo-Ruiz², Rafael Rodríguez-Ortiz ${ }^{3}$, Lenny Gallardo4, \\ Karla Itzel Aguilar-Flores 5 y Paula Cabrera-Galeana ${ }^{1 *}$ \\ ${ }^{1}$ Oncología Médica, Tumores Mamarios; ${ }^{2}$ Subdirección de Medicina Interna; ${ }^{3}$ Subdirección de Investigación Clínica, Instituto Nacional de \\ Cancerología; ${ }^{4}$ Universidad del Ejército y Fuerza Aérea; ${ }^{5}$ Hospital Ángeles Santa Mónica, Ciudad de México, México
}

\section{Resumen}

Introducción: El paclitaxel incrementa la supervivencia en cáncer de mama. Su aplicación semanal es mejor tolerada y eficaz. Uno de cada 5 pacientes tendrá neutropenia y uno de cada 15 tendrá neutropenia febril. El objetivo fue identificar factores de riesgo desarrollar neutropenia (NP) III-IV. Materiales y métodos: 257 pacientes utilizaron paclitaxel semanal con biometría hemática $(B H)$ previa a la infusión durante las 12 semanas. Se determinó edad, etapa clínica, comorbilidad, IMC, dosis total, dosis $/ \mathrm{m}^{2}$ SC, número de consultas, visitas a Urgencias y hospitalizaciones. Resultados: 118 pacientes (45.9\%) recibieron paclitaxel neoadyuvante, adyuvante $86(33.4 \%)$ y paliativo primera línea 53 pacientes (20.6\%). La frecuencia de NP III-IV fue del 5.9\%. El análisis multivariante identificó como variables independientes para NP III-IV: edad (mayores de 60 años) (OR: 3.11; IC 95\%: 1.05-9.20; $p=0.04$ ), quimioterapia previa (OR: 3.74; IC 95\%: 1.15-12.1; $p=0.027$ ), ser hipertenso (OR: 6.63; IC 95\%: 2.18-20.15; $p=0.001$ ). Conclusiones: En los pacientes mayores de 60 años, la exposición previa a quimioterapia y la hipertensión son factores de riesgo para el desarrollo de NP III-IV. La baja incidencia de NP III-IV en la práctica clínica diaria durante la aplicación de paclitaxel semanal sugiere una reconsideración de la evaluación semanal con $\mathrm{BH}$ en pacientes sin factores de riesgo.

Palabras clave: Cáncer de mama. Paclitaxel semanal. Toxicidad hematológica.

\section{Risk factors for the development of hematological toxicity during the application of weekly paclitaxel in breast cancer}

\section{Abstract}

Background: Paclitaxel increases survival in breast cancer. Weekly application is the best tolerated and most effective. One in 5 patients will have neutropenia and one in 15 patients will develop febrile neutropenia. The objective was to identify risk factors for the development of grade III-IV neutropenia. Material and methods: We retrospectively analyzed 257 patients who used weekly paclitaxel and had blood biometry before the weekly infusion for 12 weeks. We determined age, clinical stage, comorbidity, body mass index, weekly total infused dose, dose $/ \mathrm{m}^{2}$ of body surface, number of medical consultations, visits to the emergency room and hospitalizations. Results: A total of 118 patients (45.9\%) received neoadjuvant paclitaxel, adjuvant $86(33.4 \%)$ and 53 first-line palliative therapy (20.6\%). The frequency of neutropenia grade III-IV was $5.9 \%$. The multivariate analysis identified as independent factors for neutropenia grade III-IV: age (over 60 years) (OR: 3.11; Cl 95\%:

\section{Correspondencia:}

*Paula Cabrera-Galeana

E-mail: drapaulacabrera@gmail.com
Disponible en internet: 01-04-2019 Gac Mex Oncol. 2019;18:12-17 www.gamo-smeo.com 1665-9201/○ 2019 Sociedad Mexicana de Oncología. Publicado por Permanyer México SA de CV. Este es un artículo Open Access bajo la licencia CC BY-NC-ND (http://creativecommons.org/licenses/by-nc-nd/4.0/). 
1.05-9.20; $p=0.04$ ), previous chemotherapy (OR: 3.74; Cl 95\%: 1.15-12.1; $p=0.027$ ), being hypertensive (OR: 6.63; Cl 95\%: 2.18-20.15; $p=0.001$ ). Conclusions: In patients older than 60 years, exposition to previous chemotherapy and some comorbidities, particularly hypertension, are risk factors for the development of grade III-IV neutropenia. The low incidence of grade III-IV neutropenia in daily clinical practice during weekly paclitaxel suggests a reconsideration of the weekly evaluation with blood biometry in patients without risk factors.

Key words: Weekly paclitaxel. Breast cancer. Hematologic toxicity.

\section{Introducción}

El paclitaxel incrementa la supervivencia en las etapas avanzada y temprana del cáncer de mama; estabiliza y polimeriza los microtúbulos mediante su unión a la subunidad beta de la tubulina, lo que genera muerte celular durante la fase de mitosis ${ }^{1,2}$. Desde su aparición, el paclitaxel se ha evaluado en diferentes dosis, tiempos de infusión y periodos de administración. Cinco estudios prospectivos fase III han demostrado que la aplicación de forma semanal es la mejor tolerada y eficaz ${ }^{1,2}$. Sin embargo, se considera poco conveniente debido al incremento del uso de tiempos en la sala de infusión, la saturación del servicio de consulta externa, el laboratorio y el aumento de costos indirectos ${ }^{3,4}$.

La modalidad semanal en adyuvancia y neoadyuvancia se aplica durante 12 semanas consecutivas a dosis de $80 \mathrm{mg} / \mathrm{m}^{2}$ en infusión de una hora ${ }^{5}$. Se ha identificado que uno de cada 5 pacientes tendrá neutropenia y uno de cada 15 pacientes desarrollará neutropenia febril².

Pese al uso generalizado de este esquema en nuestro país, pocos estudios han evaluado la toxicidad hematológica fuera del escenario de un protocolo de investigación, cuyos datos de seguridad son difíciles de trasladar a la población no seleccionada de la práctica clínica diaria ${ }^{3}$.

No existe una recomendación clara para dar seguimiento a la toxicidad hematológica del paclitaxel semanal. Nuestro objetivo fue identificar factores de riesgo para el desarrollo de neutropenia grado III-IV y distinguir un patrón de comportamiento de la neutropenia durante las doce semanas de aplicación que permita recomendar un intervalo de evaluación para los pacientes que reciben este tipo de tratamiento.

\section{Material y métodos}

Se seleccionó una muestra retrospectiva de pacientes con diagnóstico confirmado de cáncer de mama entre los años 2011 al 2015. Se incluyó aquellos que recibieron paclitaxel semanal cuando menos 12 semanas y que contaran con una muestra de biometría hemática en cada semana de aplicación. La biometría hemática debió ser realizada en un periodo no mayor a 48 horas previa a la infusión. Se permitió la indicación de paclitaxel semanal en el escenario neoadyuvante y adyuvante, indistintamente de la secuencia con antraciclinas. En enfermedad avanzada solo se incluyeron casos en primera línea de tratamiento. Las pacientes con sobreexpresión de HER2 recibieron trastuzumab. Los datos demográficos, clínicos y de laboratorio fueron obtenidos del expediente electrónico del Instituto Nacional de Cancerología. Se identificó la edad, etapa clínica, estado de HER2, comorbilidad, el índice de masa corporal (IMC), la dosis total semanal infundida y la dosis por metro cuadrado de superficie corporal; también se registró el número de consultas médicas recibidas, visitas a la sala de emergencia y hospitalizaciones de los pacientes, durante las $12 \mathrm{se-}$ manas de paclitaxel.

Los eventos adversos hematológicos fueron clasificados según las directrices CTCAE (Common Toxicity Criteria for Adverse Events) versión 4.03. Se obtuvo el grado CTCAE más alto para cada tipo de evento adverso hematológico y para cada paciente. Las proporciones de pacientes con cualquier evento hematológico se compararon mediante la prueba de chi-cuadrada. Se evaluaron los patrones de máxima frecuencia de eventos adversos hematológicos en el tiempo. Se realizó también un análisis descriptivo de las variables demográficas. Medimos la relación de la neutropenia con factores de riesgo para su desarrollo mediante análisis univariante y multivariante.

\section{Resultados}

Se analizaron los datos de un total de 257 pacientes, cuya media de edad fue de 52 años (21 a 87 años). Etapa temprana (I-IIA), 44 casos (17.1\%); enfermedad localmente avanzada (IIB- IIIC), 161 pacientes $(62.6 \%)$; y enfermedad a distancia, 53 (20.6\%). El 22.5\% (58) pacientes tenían sobreexpresión de HER2. El paclitaxel semanal fue utilizado de forma neoadyuvante en 118 pacientes (45.9\%), como adyuvante en $86(33.4 \%)$ y como terapia paliativa de primera línea en 53 pacientes 
Tabla 1. Características demográficas y del tratamiento oncológico de los pacientes con cáncer de mama que recibieron paclitaxel semanal incluidos en el estudio

\begin{tabular}{|c|c|c|c|}
\hline Edad & Media 52 años (21-87) & $\mathrm{n}$ & $(\%)$ \\
\hline \multirow[t]{2}{*}{ IMC } & $<25$ & 74 & (28.7) \\
\hline & $>25$ & 183 & (71.2) \\
\hline \multirow[t]{5}{*}{ Comorbilidades } & DM2 & 20 & (7.7) \\
\hline & HAS & 49 & (19) \\
\hline & HAS/DM2 & 10 & (3.9) \\
\hline & Otras comorbilidades & 20 & (7.7) \\
\hline & Sin comorbilidades & 158 & (61.8) \\
\hline \multirow[t]{3}{*}{ Etapa clínica } & Temprana (I, IIA) & 44 & (17.1) \\
\hline & $\begin{array}{l}\text { Localmente avanzado } \\
\text { (IIB-IIIC) }\end{array}$ & 161 & (62.6) \\
\hline & Metastásico (IV) & 53 & (20.6) \\
\hline \multirow{2}{*}{$\begin{array}{l}\text { Modalidad de } \\
\text { tratamiento }\end{array}$} & Neoadyuvante/adyuvante & 204 & (79.4) \\
\hline & Paliativo primera línea & 53 & (20.6) \\
\hline \multirow[t]{2}{*}{ Esquema } & Paclitaxel & 199 & (77.5) \\
\hline & Paclitaxel + trastuzumab & 58 & (22.5) \\
\hline \multirow{3}{*}{$\begin{array}{l}\text { Intensidad de } \\
\text { dosis }\end{array}$} & $<75 \mathrm{mg} / \mathrm{m}^{2}$ & 27 & (10.5) \\
\hline & $75-79.9 \mathrm{mg} / \mathrm{m}^{2}$ & 177 & (68.9) \\
\hline & $\geq 80 \mathrm{mg} / \mathrm{m}^{2}$ & 53 & (20.6) \\
\hline \multirow{3}{*}{$\begin{array}{l}N .{ }^{\circ} \text { de consultas } \\
\text { médicas }\end{array}$} & $<4$ & 46 & (18.6) \\
\hline & $5-7$ & 167 & (64.9) \\
\hline & $>8$ & 44 & (17.1) \\
\hline
\end{tabular}

IMC: índice de masa corporal.

(20.6\%). El IMC, comorbilidad, número de consultas e intensidad de dosis se reportan en la tabla 1. Las toxicidades hematológicas máximas por paciente durante las doce semanas de evaluación se presentan en la tabla 2, destaca la ausencia de casos de neutropenia febril y un porcentaje menor al $1 \%$ para neutropenia, leucopenia y trombocitopenia grado IV. La frecuencia de neutropenia grado III y IV fue del $5.9 \%$. El $20 \%$ de los pacientes requirió un ajuste de dosis por toxicidad no hematológica.

Al servicio de atención inmediata acudieron 70 pacientes al menos una vez y 21 pacientes fueron hospitalizados durante el periodo de aplicación de las 12 semanas de paclitaxel. Ninguna de esas visitas y hospitalizaciones fue asociada a toxicidad hematológica.

El análisis univariante se presenta en el tabla 3. No correlacionaron con riesgo de desarrollar neutropenia grado III-IV: el IMC mayor a 25, una dosis total mayor de $140 \mathrm{mg}$ por semana, el escenario clínico de la aplicación, y el número de consultas médicas, de hospitalizaciones por otras toxicidades y de visitas a la sala de emergencia.

En el análisis multivariante de regresión logística se identificaron como variables independientes para el desarrollo de neutropenia grado III y IV: la edad (mayores de 60 años; OR: 3.11; IC 95\%: 1.05-9.20; $p=0.04$ ), el haber recibido quimioterapia previa (OR: 3.74; IC 95\%: 1.15-12.1; $p=0.027$ ) y ser hipertenso (OR: 6.63; IC 95\%: 2.18-20.15; $p=0.001$ ).

\section{Discusión}

La variabilidad de los porcentajes publicados de neutropenia grado III y IV de estudios prospectivos controlados en los tres escenarios clínicos (neoadyuvante, adyuvante y metastásico) ha generado diversas prácticas clínicas en la evaluación de la toxicidad hematológica ${ }^{5-10}$.

La frecuencia con la que debe realizarse una biometría hemática previa a la infusión semanal de paclitaxel fuera de protocolo no está descrita. En el estudio de Sparano, et al. ${ }^{5}$, que incluye 1,231 pacientes que recibieron paclitaxel semanal adyuvante, la biometría hemática se tomó cada semana previa a la infusión, por lo cual esta conducta se ha generalizado y el porcentaje de neutropenia grado III-IV fue un $2 \%$. Sin embargo, Seidman, et al. ${ }^{11}$ realizaron una biometría hemática cada tres semanas en 572 pacientes con enfermedad avanzada; la frecuencia de neutropenia grado III fue un $5 \%$ y grado IV un $3 \%$, por 10 cual algunos clínicos también utilizan esta práctica. Estos resultados contrastan con lo reportado por Ando, et al. ${ }^{12}$ quienes evaluaron 181 pacientes que recibieron paclitaxel neoadyuvante semanal seguido de antraciclinas, e identificaron un $8.8 \%$ de neutropenia grado III y un $1.1 \%$ de grado IV, mientras que en el estudio de Miller, et al. ${ }^{6}$ en 346 pacientes metastásicos que recibieron paclitaxel semanal como primera línea de tratamiento, la neutropenia grado III fue un $0.3 \%$ y ningún paciente presentó neutropenia grado IV. Resulta difícil determinar un riesgo exacto de toxicidad hematológica.

Nuestro estudio reporta una frecuencia de neutropenia grado III del $5.1 \%$ y grado IV del $0.4 \%$ en pacientes no seleccionados de la clínica habitual; estos 
Tabla 2. Toxicidad hematológica por paciente durante las 12 semanas de aplicación de paclitaxel semanal en pacientes con cáncer de mama evaluados en el estudio

\begin{tabular}{|l|c|c|c|c|c|}
\hline Toxicidad & $\begin{array}{c}\text { Grado I } \\
\mathbf{n}(\%)\end{array}$ & $\begin{array}{c}\text { Grado II } \\
\mathbf{n}(\%)\end{array}$ & $\begin{array}{c}\text { Grado III } \\
\mathbf{n}(\%)\end{array}$ & $\begin{array}{c}\text { Grado IV } \\
\mathbf{n}(\%)\end{array}$ & $\begin{array}{c}\text { Sin toxicidad } \\
\text { n (\%) }\end{array}$ \\
\hline Neutropenia & $47(18.3)$ & $29(11.3)$ & $13(5.1)$ & $1(0.4)$ & $167(65)$ \\
\hline Leucopenia & $97(37.7)$ & $42(16.3)$ & $11(4.3)$ & $2(0.8)$ & $105(40.9)$ \\
\hline Anemia & $85(33.1)$ & $18(7.0)$ & $1(0.4)$ & $0(0)$ & $153(59.5)$ \\
\hline Trombocitopenia & $27(10.5)$ & $6(2.3)$ & $0(0)$ & $0(0)$ & $224(87.2)$ \\
\hline Linfopenia & $93(36.2)$ & $41(16)$ & $10(3.9)$ & $4(1.6)$ & $109(42.4)$ \\
\hline
\end{tabular}

Tabla 3. Análisis univariante para evaluar los factores de riesgo para el desarrollo de toxicidad hematológica durante la aplicación de paclitaxel semanal

\begin{tabular}{|c|c|c|c|}
\hline Variable & OR & $\begin{array}{c}\text { Intervalo de } \\
\text { confianza } 95 \%\end{array}$ & $\mathbf{p}$ \\
\hline Edad $<60$ años & 0.256 & $0.103-0.633$ & 0.04 \\
\hline Edad $>60$ años & 4.453 & $1.632-12.149$ & 0.04 \\
\hline IMC $>25$ & 0.818 & $0.278-2.402$ & NS \\
\hline Dosis total mayor de $140 \mathrm{mg}$ & 1.677 & $0.188-2.440$ & NS \\
\hline $\begin{array}{l}\text { Pacientes con }>8 \text { consultas } \\
\text { médicas }\end{array}$ & 0.473 & $0.176-1.273$ & NS \\
\hline Quimioterapia previa & 1.442 & $1.144-8.810$ & 0.01 \\
\hline $\begin{array}{l}\text { Tratamiento neoadyuvante } 0 \\
\text { adyuvante }\end{array}$ & 1.11 & $0.810-1.520$ & NS \\
\hline Tratamiento paliativo $1 .^{a}$ línea & 0.743 & $0.340-1.610$ & NS \\
\hline $\begin{array}{l}\text { Tener comorbilidad } \\
\text { (DM, obesidad, HAS) }\end{array}$ & 6.419 & 2.027-20.322 & 0.04 \\
\hline Hipertensión arterial sistémica & 5.805 & $2.099-16.055$ & 0.01 \\
\hline & & & NS \\
\hline Hospitalización & 0.681 & $0.086-5.407$ & NS \\
\hline
\end{tabular}

IMC: índice de masa corporal.

porcentajes confirman la seguridad hematológica de la aplicación semanal del paclitaxel en nuestra práctica diaria. El resultado es similar al $5.7 \%$ de neutropenia grado III-IV referido en población brasileña expuesta a paclitaxel semanal, fuera de protocolo clínico ${ }^{3}$.

El porcentaje de pacientes que tienen reducción de dosis al utilizar este esquema se ha reportado para enfermedad metastásica en un 27 y un $29 \%$ de los pacientes en adyuvancia ${ }^{5,12,13}$, siendo la principal toxicidad limitante de dosis la neurotoxicidad ${ }^{2}$. En nuestros datos se identificó una reducción de dosis en el $20 \%$ de los pacientes debido a toxicidades no hematológicas; este valor puede estar influido por las características de la población, que incluye pacientes en adyuvancia, neoadyuvancia y terapia paliativa de primera línea. Por otro lado, el $89.5 \%$ de la población mantuvo una intensidad de dosis arriba de $75 \mathrm{mg} / \mathrm{m}^{2} / \mathrm{semana}$ pese a que 1 de cada 5 pacientes tenía enfermedad avanzada, comparable con la media de intensidad de dosis reportada fuera de protocolo clínico de $77 \mathrm{mg} / \mathrm{m}^{2} / \mathrm{semana}$ para enfermedad temprana ${ }^{3}$. De acuerdo a una publicación previa de nuestro grupo, este $10.5 \%$ de pacientes con intensidad de dosis menor a $75 \mathrm{mg} / \mathrm{m}^{2} /$ semana no impactan negativamente en la supervivencia de nuestra población ${ }^{14}$.

No encontramos estudios previos que identifiquen factores de riesgo para el desarrollo de toxicidad hematológica durante la administración de paclitaxel semanal. En el análisis multivariante nuestros resultados muestran claramente tres factores independientes para el desarrollo de neutropenia grado III y IV: edad mayor de 60 años, presencia de hipertensión y la exposición previa del paciente a algún tipo de quimioterapia. Al igual que nosotros, Lichtman, et al. refieren que los pacientes mayores de 65 años presentan mayor riesgo de desarrollar toxicidad hematológica asociada a paclitaxel semanal, y este riesgo también se vio incrementado si los pacientes habían recibido previamente quimioterapia ${ }^{15}$. Por su parte Barcenas, et al. determinaron que las pacientes mayores que utilizaron doxorubicina/ciclofosfamida seguido de paclitaxel semanal durante la adyuvancia tuvieron una OR de 1.80 (1.18-2.76) para hospitalización secundaria a quimioterapia; describieron también que un índice Charlson mayor a 2 puntos 
confirió una OR de 2.70 (2.03-3.59). Si bien dichas hospitalizaciones no incluyen solo toxicidad hematológica, fortalecen nuestros resultados al establecer a la edad, la comorbilidad y la quimioterapia previa como factores asociados a toxicidad hematológica por quimioterapia ${ }^{16}$.

Destaca en nuestro estudio la OR de 6.63 (IC 95\%: 2.18-20.15; $p=0.001$ ) para hipertensión. Si bien no ha sido reportado previamente, el fenómeno puede ser explicado porque un $38.2 \%$ de pacientes analizados tenían algún tipo de comorbilidad y de ellos el 59.5\% tenían hipertensión, sola o asociada a otra comorbilidad (DM2/obesidad).

La naturaleza retrospectiva del estudio le confiere limitaciones: en protocolos prospectivos el uso de factor estimulante de colonias se encuentra permitido, no contamos con el dato exacto del uso de factor estimulante de colonias utilizado en nuestra población. Lo que podría explicar que no identificamos casos con neutropenia febril, pese a que las frecuencias publicadas son desde el 0.1 al $1 \%$ cuando el paclitaxel semanal es utilizado en adyuvancia ${ }^{5,8}$. La heterogeneidad en el manejo de los eventos adversos hematológicos de los clínicos no se documentó, por lo que al igual que otros estudios retrospectivos tenemos un sesgo de registro y de selección, ya que la decisión de indicar y administrar el tratamiento no fue controlada ${ }^{9}$.

Uno de nuestros objetivos fue reconocer un patrón de comportamiento de neutropenia, pero su baja incidencia impidió su alcance, por lo que no es posible hacer recomendaciones sobre el momento ideal para realizar una biometría hemática para evaluar la toxicidad hematológica de forma general.

Sin embargo, dado que la mayoría de los servicios de oncología de nuestro país se encuentran saturados, consideramos que identificar un grupo de alto riesgo podría ayudar a optimizar los recursos humanos y de laboratorio. Ya que incluso cuando el paclitaxel semanal es utilizado sin previa exposición a quimioterapia, los porcentajes de neutropenia grado III-IV bajan a 3.0 y $4.2 \%{ }^{17,18}$. Realizar una biometría hemática cada tres semanas nos parece una buena práctica en ausencia de factores de riesgo, ya que el estudio de Seidman que incluyó pacientes con enfermedad avanzada registró un porcentaje de neutropenia grado III-IV del 8 y el $65 \%$ de los pacientes habían recibido quimioterapia previamente $^{11}$.

Los hallazgos de nuestro estudio podrían contribuir a implementar estrategias de priorización para liberar espacios en la consulta oncológica, la toma de muestras de laboratorio y disminuir costos directos e indirectos, ya que la aplicación de paclitaxel semanal incrementa los costos de atención ${ }^{4}$.

\section{Conclusiones}

Los factores de riesgo para desarrollar neutropenia grado III-IV son: edad mayor de 60 años, exposición previa a quimioterapia, comorbilidad e hipertensión. Este grupo de pacientes se beneficia de una vigilancia hematológica estrecha. La probabilidad de tener efectos adversos hematológicos secundarios a la aplicación de paclitaxel semanal en la práctica clínica diaria es baja. La evaluación semanal con biometría hemática podría reconsiderarse. Se recomienda una evaluación con biometría cada tres semanas en ausencia de dichos factores.

\section{Agradecimientos}

Los autores expresan su agradecimiento a los doctores: Rebeca Ramírez, Jorge Alegría, Josana Rodríguez, Claudia Arce, Fernando Lara y Manuel Magallanes.

\section{Bibliografía}

1. von Minckwitz G, Martin M, Wilson G, Alba E, Schmidt M, Biganzoli L, et al. Optimizing taxane use in $\mathrm{MBC}$ in the emerging era of targeted chemotherapy. Crit Rev Oncol Hematol. 2013;85(3):315-31.

2. Carbognin L, Sperduti I, Nortilli R, Brunelli M, Vicentini C, Pellini F, et al. Balancing activity and tolerability of neoadjuvant paclitaxel- and docetaxel-based chemotherapy for HER2-positive early stage breast cancer: Sensitivity analysis of randomized trials. Cancer Treat Rev. 2015;41(3): 262-70.

3. Santana IA, Oliveira JA, da Silva Lima JM, Testa L, Piato JR, Hoff PM, et al. Feasibility of two schedules of weekly paclitaxel in HER2-negative early breast cancer in a Brazilian community setting. Breast Cancer. 2016;23(2):261-5.

4. Frías C, Cortés J, Seguí MÁ, Oyagüez I, Casado MÁ. Cost-effectiveness analyses of docetaxel versus paclitaxel once weekly in patients with metastatic breast cancer in progression following anthracycline chemotherapy, in Spain. Clin Transl Oncol. 2010;12:692-700.

5. Sparano JA, Wang M, Martino S, Jones V, Perez EA, Saphner T, et al. Weekly paclitaxel in the adjuvant treatment of breast cancer. $\mathrm{N}$ Engl $\mathrm{J}$ Med. 2008;358(16):1663-71.

6. Miller K, Wang M, Gralow J, Dickler M, Cobleigh M, Perez EA, et al. Paclitaxel plus bevacizumab versus paclitaxel alone for metastatic breast cancer. N Engl J Med. 2007;357(26):2666-76.

7. Qi WX, Shen Z, Lin F, Sun YJ, Min DL, Tang LN, et al. Paclitaxel-based versus docetaxel-based regimens in metastatic breast cancer: a systematic review and meta-analysis of randomized controlled trials. Curr Med Res Opin. 2013;29(2):117-25.

8. Budd GT, Barlow WE, Moore HCF, Hobday TJ, Stewart JA, Isaacs C et al. SWOG S0221: A phase III trial comparing chemotherapy schedules in high-risk early-stage breast cancer. J Clin Oncol. 2015;33(1): 58-64.

9. Alsharedi M, Gress T, Dotson J, Elmsherghi N, Tirona MT. Comparison of toxicity profile and tolerability between two standard of care paclitaxel-based adjuvant chemotherapy regimens in breast cancer. Med Oncol. 2016;33(3):1-5.

10. Gonzalez-Angulo AM, Akcakanat A, Liu S, Green MC, Murray JL, Chen $\mathrm{H}$, et al. Open-label randomized clinical trial of standard neoad- 
juvant chemotherapy with paclitaxel followed by FEC versus the combination of paclitaxel and everolimus followed by FEC in women with triple receptor-negative breast cancer. Ann Oncol. 2014;25(6): 1122-7.

11. Seidman $A D$, Berry D, Cirrincione $C$, et al. Randomized phase III trial of weekly compared with every-3-weeks paclitaxel for metastatic breast cancer, with trastuzumab for all HER-2 overexpressors and random assignment to trastuzumab or not in HER-2 nonoverexpressors: Final results of cancer and leu. J Clin Oncol. 2008;26(10):1642-9.

12. Ando M, Yamauchi H, Aogi K, et al. Randomized phase II study of weekly paclitaxel with and without carboplatin followed by cyclophosphamide/ epirubicin/5-fluorouracil as neoadjuvant chemotherapy for stage II/IIIA breast cancer without HER2 overexpression. Breast Cancer Res Treat. 2014;145(2):401-9.

13. John $M$, Hinke $A$, Stauch $M$, et al. Weekly paclitaxel plus trastuzumab in metastatic breast cancer pretreated with anthracyclines-a phase II multipractice study. BMC Cancer. 2012;12(1):165
14. Reynoso-Noverón N, Villareal-Garza C, Soto-Perez-de-Celis E, Arce-Salinas C, Matus-Santos J, Ramírez-Ugalde MT, et al. Clinical and epidemiological profile of breast cancer in Mexico, results of the Seguro Popular. J Glob Oncol. 2017:3(6):757-64.

15. Lichtman SM, Hurria A, Cirrincione CT, et al. Paclitaxel efficacy and toxicity in older women with metastatic breast cancer: Combined analysis of calgb 9342 and 9840. Ann Oncol. 2012;23(3):632-8.

16. Barcenas $\mathrm{CH}$, Niu J, Zhang N, et al. Risk of hospitalization according to chemotherapy regimen in early-stage breast cancer. J Clin Oncol. 2014; 32(19):2010-7.

17. Tolaney SM, Barry WT, Dang CT, et al. Adjuvant paclitaxel and trastuzumab for node-negative, HER2-positive breast cancer. N Engl J Med. 2015;372(2): 134-41.

18. Shulman LN, Berry DA, Cirrincione CT, et al. Comparison of doxorubicin and cyclophosphamide versus single-agent paclitaxel as adjuvant therapy for breast cancer in women with 0 to 3 positive axillary nodes: CALGB 40101 (alliance). J Clin Oncol. 2014;32(22):2311-7. 